\title{
Metabolism of Progesterone in the Hepatopancreas of Whelk, Buccinum undatum
}

\author{
Shin-ichi Teshima and Akio Kanazawa* \\ (Received November 20, 1970)
}

\begin{abstract}
In the present study, the in vitro bioconversion of progesterone by the minces of hepatopancreas of whelk, Buccinum undatum, was investigated. After the incubation of progesterone-4-14 $\mathrm{C}$ with the hepatopancreas, radioactive testosterone, $17 \alpha$-hydroxyprogesterone, deoxycorticosterone, and androstenedione were obtained. The identification of these metabolites were based on the mobilities on thin-layer and paper chromatography and on the chemical characteristics in the derivative formation.

The results suggested that the hepatopancreas of whelk, Buccinum undatum, may contain steroid $17 \alpha$-hydroxylase, steroid 21 -hydroxylase, steroid $C_{17}-C_{21}$ lyase, and steroid $17 \beta-$ hydroxy steroid dehydrogenase.
\end{abstract}

In fish, the metabolism of steroid hormones has been studied within the last decade ${ }^{1)}$. During the course of these investigations, 11-ketotestosterone ${ }^{2-4)}$ and $1 \alpha$-hydroxycorticosterone ${ }^{5,6)}$ were isolated as a new steroid hormones from the teleosts and elasmobranches, respectively. These findings suggest strongly that the steroidogenesis in marine animals is not necessarily similar to that of higher animals. In the previous papers, the authors reported on the transformations of progesterone by the ovaries ${ }^{7)}$ and testes ${ }^{8)}$ of the crab, Portumus trituberculatus. The purpose of the present investigation is to clarify the enzyme systems for the steroidogenesis in the hepatopancreas of the whelk.

\section{Experimental and Results}

The whelk, Buccinum undatum, was obtained alive from the commercial source, in December, 1969. The hepatopancreas of this animal was subjected to incubation with progesterone-4- ${ }^{14} \mathrm{C}$. The details of the procedures for incubation were described in the previous paper. ${ }^{7)}$

The minced hepatopancreas $(470 \mathrm{mg}$ ) was preincubated in $8 \mathrm{ml}$ of the incubation medium $^{9)}$ containing cofactors $(1.25 \mathrm{mg}$ of nicotinamide-adenine dinucleotide phosphate, $5 \mathrm{mg}$ of glucose-6-phosphate, and 1 unit of glucose-6-phosphate dehydrogenase) and antibiotics ( 9000 units of penicillin $\mathrm{G}$ and $250 \mu \mathrm{g}$ of aureomycin) at $20^{\circ} \mathrm{C}$ for 30 minutes, and then the medium was changed with fresh one. To this, $1 \mu \mathrm{Ci}$ of progesterone- $4-{ }^{14} \mathrm{C}$ $(29.3 \mathrm{mCi} / \mathrm{mM})$, dissolved in $0.01 \mathrm{ml}$ of ethanol-propylene glycol $(1: 1)$, was added and incubated for 6 hours successively. The medium was refreshed every 2 hours. After

* Faculty of Fisheries, Kagoshima University. 470 Shimoarata-cho, Kagoshima City (f

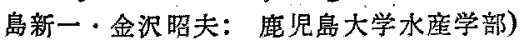


the incubation, the metabolites were extracted 3 times with 7 volumes of dichloromethane, washed with distilled water, and then the solvent was evaporated under reduced pressure.

To the dichloromethane extract, about $50 \mu \mathrm{g}$ each of non-radioactive cortisol, cortisone, corticosterone, 11-deoxycortisol, 11-ketotestosterone, 11 $\beta$-hydroxyandrostenedione, $17 \alpha$-hydroxyprogesterone, deoxycorticosterone, androstenedione, and testosterone were added as a carrier, and then chromatographed initially on thin-layer (Kieselgel G-Kieselgel $\left.\mathrm{GF}_{254}(5: 1)\right)$ using chloroform-methanol (97:3) as a solvent. The results are shown in Table 1. The materials $(E-8,9,10$, and 11) revealing both UV-absorption and high radioactivities were eluted from the plate with dichloromethane-methanol $(9: 1)^{10}$; and subjected to the investigation of metabolites by thin-layer chromatography (TLC), 11 and by paper chromatography (PPC) ${ }^{12)}$. The solvent systems used were: S1, chloroformmethanol (97:3); S2, chloroform-methanol-water (188:12:1); S3, dichloromethanemethanol $(9: 1)$; S4, benzene-acetone $(8: 2)$; S5, benzene-acetone $(75: 25)$; S6, ethyl acetatecyclohexane-toluene (10:10:1); HBM-70, heptane-benzene (1:1)-70\% methanol. Finally, for the purpose of confirming the identification of metabolites, the chromatographically purified metabolites were subjected to the acetylation by acetic acid anhydridepyridine $(1: 1)^{7)}$ and/or to the oxidation by $0.5 \% \mathrm{CrO}_{3}$ in $90 \%$ acetic acid, $\left.{ }^{7}\right)$ and then the formed derivatives were investigated.

Table 1. Initial TLC of the dichloromethane extract of the metabolites from progesterone${ }^{4-14} \mathrm{C}$.

\begin{tabular}{c|c|c|c}
\hline \hline Eluates & Rf & UV-absorption & Radioactivity (dpm) \\
\hline E-1 & $0.00-0.03$ & - & 2620 \\
E-2 & $0.03-0.06$ & + & 2700 \\
E-3 & $0.06-0.10$ & + & 2160 \\
E-4 & $0.10-0.13$ & + & 3850 \\
E-5 & $0.13-0.20$ & + & 4000 \\
E-6 & $0.20-0.24$ & + & 4160 \\
E-7 & $0.24-0.26$ & - & 6310 \\
E-8 & $0.26-0.33$ & + & 10200 \\
E-9 & $0.33-0.51$ & + & 31700 \\
E-10 & $0.51-0.59$ & + & 8930 \\
E-11 & $0.59-0.73$ & + & 564000 \\
E-12 & $0.73-0.77$ & - & 19300 \\
E-13 & $0.77-0.83$ & - & 13200 \\
E-14 & $0.83-1.00$ & - & 2310 \\
\hline
\end{tabular}

The material (E-8) corresponding to testosterone and $11 \beta$-hydroxyandrostenedione in the initial TLC was rechromatographed on TLC in systems S1, S2, and on PPC in system HBM-70, successively. In these chromatography, the material gave one spot showing the high radioactivites. When this substance was chromatographed on TLC 
in system 6 , the two radioactive materials exhibiting the same mobilities as $11 \beta$-hydroxyandrostenedione (Rf, 0.17) and testosterone ( $\mathrm{Rf}, 0.25$ ) were detected. The presumed $11 \beta$-hydroxyandrostenedione ${ }^{14} \mathrm{C}$ was oxidized with $0.5 \% \mathrm{CrO}_{3}$ in $90 \%$ acetic acid. The oxidation product revealed the same mobility as authentic adrenosterone in TLC using system S4, although a significant radioactivity was not demonstrated in this material. The oxidation and acetylation of the assumed testosterone $-{ }^{14} \mathrm{C}$ gave the radioactive androstenedione and testosterone $17 \beta$-mono acetate, respectively.

The material (E-9) corresponding to $17 \alpha$-hydroxyprogesterone was rechromatographed on TLC in systems S4, S3, S6 and on PPC in system HBM-70, successively. Throughout these purification steps, this substance showed relatively high radioactivites. When the chromatographically purified material was subjected to oxidation and acetylation, the sutstances showing the same mobilities as authentic androstenedione and $17 \alpha$ hydroxyprogesterone were obtained respectively.

The material $(E-10)$ corresponding to deoxycorticosterone was rechromatographed on TLC in system S3 and on PPC in system HBM-70, successively. The acetylation of this substance gave deoxycorticosterone 21-mono acetate.

The material (E-11) corresponding to androstenedione and progesterone (precursor) was rechromatographed on TLC in system S5. In this system, androstenedione (Rf, 0.34 ) was separated from progesterone ( $\mathrm{Rf}, 0.65$ ). The material corresponding to androstenedione was rechromatographed on TLC in system S3, S6, and on PPC in system HBM-70, successively. In these chromatography, this substance revealed a significant radioactivities and the same mobilities as standard androstenedione. Furthermore, the material was not acetylated with acetic acid anhydride-pyridine $(1: 1)$.

Table 2. Derivative formations of the metabolites from progesterone $-4-{ }^{14} \mathrm{C}$ by the hepatopancreas of the whelk.

\begin{tabular}{|c|c|c|c|}
\hline \multirow{2}{*}{ Metabolites } & \multirow{2}{*}{ Reactions } & \multicolumn{2}{|c|}{${ }^{14} \mathrm{C}$-Derivatives by chemical reactions } \\
\hline & & $\mathrm{Rf}$ in $\mathrm{TLC}^{*}$ & Identified as \\
\hline Testosterone- ${ }^{-14} \mathrm{C}$ & $\begin{array}{l}\text { Acetylation } \\
\text { Oxidation }\end{array}$ & $\begin{array}{l}0.56 \\
0.44\end{array}$ & $\begin{array}{l}\text { Testosterone } 17 \beta \text {-mono acetate } \\
\text { Androstenedione }\end{array}$ \\
\hline $17 \alpha$-Hydroxyprogesterone-14 C & $\begin{array}{l}\text { Acetylation } \\
\text { Oxidation }\end{array}$ & $\begin{array}{l}0.36 \\
0.45\end{array}$ & $\begin{array}{l}17 \alpha \text {-Hydroxyprogesterone } \\
\text { Androstenedione }\end{array}$ \\
\hline Deoxycorticosterone- ${ }^{14} \mathrm{C}$ & Acetylation & 0.52 & Deoxycorticosterone 21-mono acetate \\
\hline Androstenedione- ${ }^{14} \mathrm{C}$ & Acetylation & 0.44 & Androstenedione \\
\hline
\end{tabular}

* Calculated from the thin-layer chromatogram developed by system $\mathbf{S 4}$.

\section{Discussion}

In the present study, the minced hepatophancreas of the whelk was incubated with 
progesterone $-4-{ }^{14} \mathrm{C}$. As the metabolites of progesterone, $17 \alpha$-hydroxyprogesterone, androstenedione, testosterone, and deoxycorticosterone were tentatively identified by chromatography and by derivative formation. However, the low activities in the materials did not allow to confirm the identification on the basis of constant specific activity (dpm/ $\mathrm{mg}$ ) during repeated crystallizations with authentic steroids.

As the possible formation steps of the above metabolites, the following pathways are considerable:

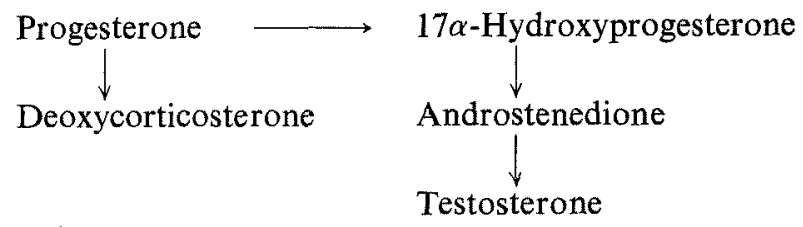

From these results, therefore, it is suggested that the hepatopancreas of the whelk, Buccinum undatum, may possess the enzyme systems for $17 \alpha$-hydroxylation, 21-hydroxylation, side-chain cleavage, and $17 \beta$-dehydrogenation.

In marine invertebrates, the function of hepatopancreas on the metabolism of steroid hormones scarcely has been clarified. TCHOLAKIAN and EIK-NES reported that progesterone is transformed to deoxycorticosterone by the hepatopancreas homogenate of the blue crab, Callinectes sapidus, (conversion rate, $0.3 \%$ ), although the enzyme activity is low in comparison with that of the androgenic gland (conversion rate, 6.2\%). ${ }^{13}$ However, the details of identification procedure for deoxycorticosterone on the hepatopancreas were not shown in the paper. On the other hand, IDLER et al., demonstrated that the sliced gonadal tissues of the scallop, Placopecten magellanicus, converted $17 \alpha$-hydroxyprogesterone to androstenedione, and that the enzymic activity for side-chain cleavage of $17 \alpha$ hydroxyprogesterone was not shown in the hepatopancreas. ${ }^{14)}$

\section{Acknowledgement}

The authors wish to thank Prof. K. Kashiwada, University of Kagoshima, for his kind advice and encouragement during the present study.

\section{References}

1) R. Arai and N. Egami: Hormones and Clinics. 16, 97-112 (1968).

2) D. R. Idler, P. J. Schmidr, and A. P. Ronald: Can. J. Biochem. Physiol., 38, 1053-1057 (1960).

3) P. J. SchMid and D. R. Idler: Gen. Comp. Endocrinol,, 2, 204-214 (1962).

4) U. EYlath and B. Eckstern: ibid., 12, 58-62 (1969).

5) D. R. Idler and B. Truscott: J. Fish. Res. Bd. Can., 23, 615-619 (1966).

6) B. Truscott and D. R. IdLeR: J. Endocrinol., 40, 515-526 (1968).

7) S. Teshima and A. Kanazawa: Gen. Comp. Endocrinol, in press.

8) S. Teshima and A. Kanazawa: This Bull., 37, 524-528 (1971). 
9) S. Teshima and A. Kanazawa: ibid., 36, 246-249 (1970).

10) D. R. Idler, N. R. Kimball, and B. Truscott: Steroids, 8, 865-876 (1966).

11) S. HARA and M. TAKEUCHI: Endocrinol. Japan, 10, 202-207 (1963).

12) I. E. Bush: The Chromatography of Steroids, MacMillan (Pergamon), New York (1961).

13) R. K. Tcholakian and K. B. Eik-Nes: Gen. Comp. Endocrinol., 12, 171-173 (1969).

14) D. R. Idler, G. B. Sangalang, and A. Kanazawa: ibid., 12, 222-230 (1969). 